\title{
Actinomycosis of the parotid gland: case series
}

\author{
Dong Hoon Lee, Eun Kyung Jung, Joon Kyoo Lee, Sang Chul Lim
}

Department of Otolaryngology-Head and Neck Surgery, Chonnam National University Medical School \& Hwasun Hospital, Hwasun, South Korea Contributions: (I) Conception and design: DH Lee; (II) Administrative support: DH Lee; (III) Provision of study materials or patients: All authors; (IV) Collection and assembly of data: All authors; (V) Data analysis and interpretation: DH Lee; (VI) Manuscript writing: All authors; (VII) Final approval of manuscript: All authors.

Correspondence to: Dong Hoon Lee, MD, PhD. Department of Otolaryngology-Head and Neck Surgery, Chonnam National University Medical School and Hwasun Hospital, 160 Ilsimri, Hwasun, Jeonnam, 519-809, South Korea. Email: Leen3L@hanmail.net.

\begin{abstract}
Background Actinomycosis of the parotid gland is very rare. We sought to examine the clinical features, treatment methods, and treatment results of actinomycosis of the parotid gland in our hospital.

Methods: We retrospectively enrolled 5 patients with histopathologically identified actinomycosis of the parotid gland from January 2010 to May 2020.

Results: This study included 3 male and 2 female subjects. All patients had a common complaint of the mass in the parotid gland. Skin necrosis was observed in one patient. However, skin necrosis and fistula track occurred in the other 2 patients after fine-needle aspiration cytology (FNAC). Based on the result of FNAC two cases of inflammation and abscess were identified. Four patients with suspicion of parotid tumors before surgery underwent parotidectomy, and one patient with consideration of parotid abscess underwent incision and drainage. After surgery, intravenous administration of antibiotics was performed for an average of 6 days and oral antibiotics were prescribed for about 2 months for patients with actinomycosis of the parotid gland.

Conclusions: In case of skin necrosis of the parotid lesion without evidence of a malignant tumor, or if FNAC does not reveal the presence of cancerous cells, and fistula tract or skin necrosis occurs after the FNAC, then actinomycosis of the parotid gland should be suspected. If the actinomycosis of the parotid gland was localized to the parotid gland and removed surgically, it would be sufficient to treat the patients with antibiotics for about 2 months while observing the clinical response.
\end{abstract}

Keywords: Parotid gland; actinomycosis; antibiotics; surgical procedures; fine-needle biopsy

Submitted Mar 30, 2021. Accepted for publication Apr 14, 2021.

doi: $10.21037 /$ apm-21-755

View this article at: http://dx.doi.org/10.21037/apm-21-755

\section{Introduction}

Actinomycosis is a rare granulomatous lesion characterized by the formation abscess, fibrosis of tissues, and draining sinuses, usually caused by Actinomyces israelii (1-6). Actinomycosis is classified according to the anatomical site infected as cervicofacial ( $55 \%$ of cases, most common), thoracic, abdominopelvic, and central nervous system, of which cervicofacial is the most common type $(1,2,4,5)$. However, parotid actinomycosis is very rare, so only a few cases have been reported since Leonarmant first reported primary actinomycosis of parotid gland in 1913
(2-9). Actinomycosis requires a differential diagnosis from parotid lesions, including benign tumor, carcinoma, tuberculosis, cellulitis, fungal infections, and pyogenic abscess (2-4,6-9). The main treatment for actinomycosis is antibiotic therapy, but the exact duration of treatment has not been established (1-11).

Therefore, we sought to examine the clinical features, treatment methods, and treatment results of actinomycosis of the parotid gland in our hospital.

We present the following article in accordance with the AME Case Series Checklist (available at http://dx.doi. org/10.21037/apm-21-755). 


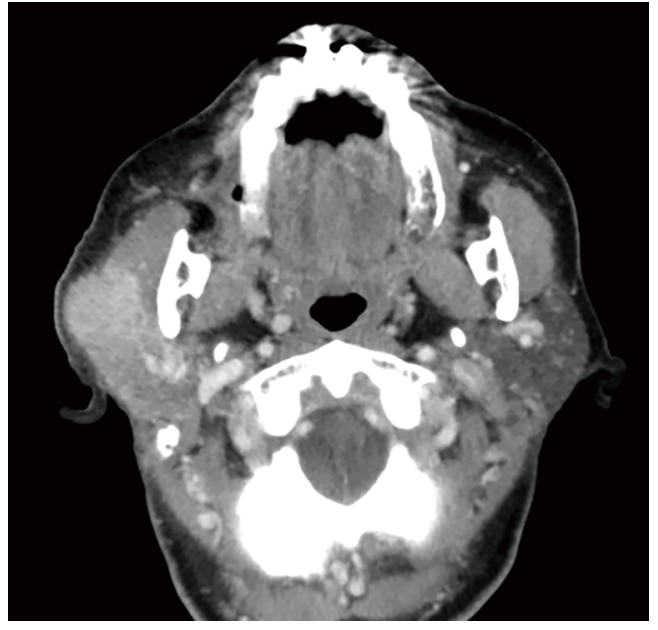

Figure 1 A 72-year-old female patient with actinomycosis of the parotid gland. Neck CT scan shows a $3 \mathrm{~cm} \times 2.5 \mathrm{~cm} \times 3.3 \mathrm{~cm}$ heterogeneously enhancing mass with perilesional infiltration in the right parotid gland.

\section{Methods}

All patient samples were treated in accordance with the Declaration of Helsinki (as revised in 2013), and the study was approved by the independent ethics committees at Chonnam National University Hwasun Hospital (CNUHH-2020-141), written informed consent was obtained from the patient. A review of 968 patients who underwent parotid surgery at this hospital for about 11 years from 2010 to 2020 confirmed 5 patients $(5 / 968$, $0.005 \%$ ) diagnosed with actinomycosis in the final biopsy after surgery. The patients' clinical data were reviewed for age, sex, underlying diseases, a dental problem, trauma, previous infection, location, symptoms, duration of symptoms, fine-needle aspiration cytology (FNAC), diagnostic method, skin status, preoperative diagnosis, surgical procedures, microbiological culture, postoperative treatment, complications, and recurrence.

All patients underwent computed tomography (CT) before surgery for the assessment of the extent of the lesions and to aid in treatment planning (Figure 1). Preoperative skin status was divided into intact and necrosis. All patients underwent surgery, and the histopathological examination confirmed actinomycosis.

\section{Statistical analysis}

The results were presented as median and range as well as mean $\pm \mathrm{SD}$. The quantitative data were analyzed with Student's $t$-test using Statistical Package for the Social Sciences version 20.0 (IBM Corporation, Armonk, NY, USA). $\mathrm{P}<0.05$ was considered a statistically significant value.

\section{Results}

The clinical data of the five patients with actinomycosis of the parotid gland are summarized in Table 1. This study included 3 male $(60.0 \%)$ and 2 female $(40.0 \%)$ subjects. The age of patients ranged between 21 and 74 years, with a mean of $56.4 \pm 21.4$ years. Only one patient had hypertension as the underlying disease, and the other four had no previous medical problems. The patients did not undergo any dental treatment recently and had no complaints of dental abnormalities such as discomfort in their teeth. One patient underwent a facelift procedure at the same site a year before the onset of the disease. Two patients had swelling or pain in the same area 3 months back.

All patients complained of the parotid mass as the main symptom. The duration of the symptom was $1.4 \pm$ 1.5 months (range, 0.3 to 4 months). Among the five lesions, four $(80.0 \%)$ were in the right parotid gland and one $(20.0 \%)$ was in the left gland. The size of the lesion was $2.8 \pm 1.2 \mathrm{~cm}$ (range, 1.2 to $4.4 \mathrm{~cm}$ ). The preoperative skin status was mainly intact $(n=4)$. Necrosis was observed in one patient. However, skin necrosis occurred in 2 of 4 FNAC patients. Based on the result of FNAC two cases of inflammation and abscess were identified

Four patients with suspected parotid tumor prior to surgery underwent superficial parotidectomy with facial nerve preservation (Figure 2), and one patient with probable presence of parotid abscess underwent incision and drainage. There was no major surgical complications including, facial nerve palsy. The culture test in 3 patients revealed the absence of actinomycosis. After surgery, mainly cephalosporin was administered intravenously for an average of 6 days (range, 5 to 9 days). In one patient, initially cephalosporin was administered and later replaced with penicillin after the diagnosis of actinomycosis based on the recommendation of the Department of Infectious Diseases. Subsequently, oral penicillin was prescribed for an average of 57.8 days (range, 28 to 105 days). The duration or dose of antibiotic treatment was determined in consultation with the Department of Infectious Diseases after checking the severity of the disease and the patient's symptoms.

The mean follow-up period after surgery was $40.0 \pm$ 31.2 months, with a range from 1 to 76 months. One 


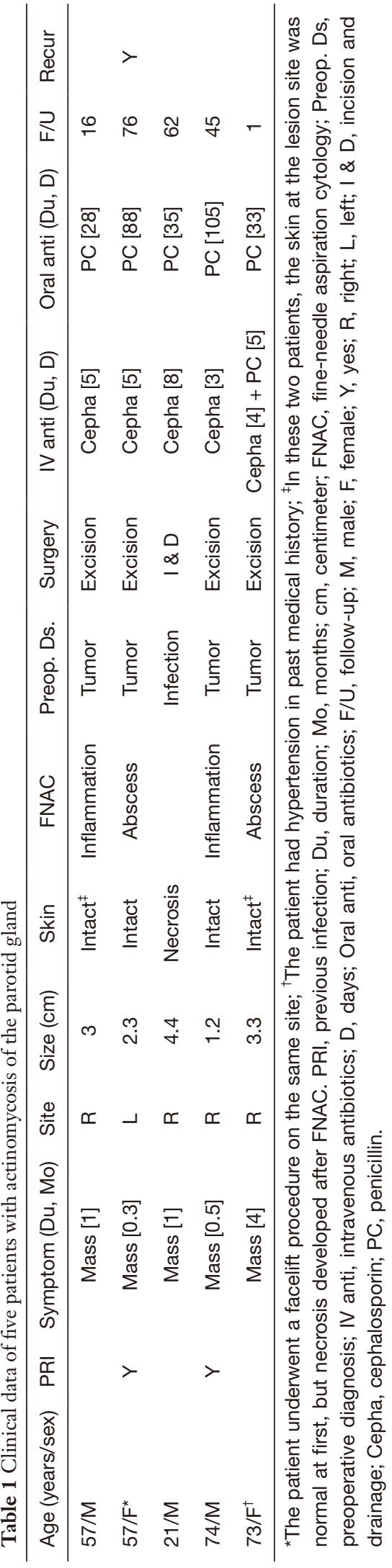

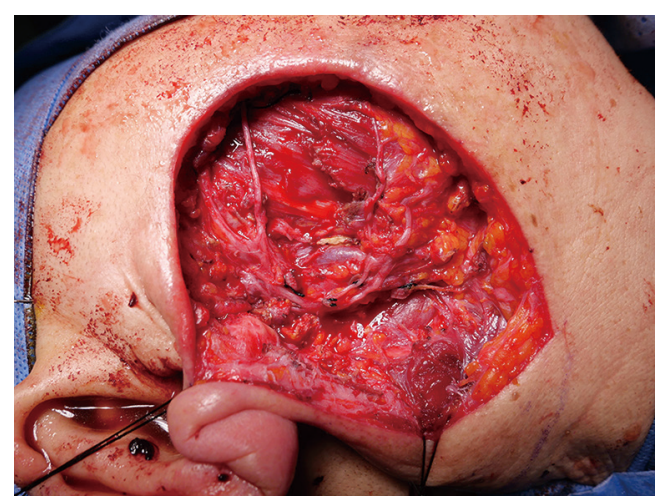

Figure 2 A 72-year-old female patient with actinomycosis of the parotid gland. The intraoperative photograph demonstrates that parotid actinomycosis has been removed cleanly.

patient had relapsed after two years of completion of the first treatment and demonstrated improvement with antibiotic treatment for two weeks. Since then, there have been no cases of relapse.

\section{Discussion}

Five patients with complaints of actinomycosis of the parotid gland for the past 10 years were mainly diagnosed with parotid tumors before surgery. Consequently, the characteristics of the actinomycosis of the parotid gland are discussed in the present study.

Actinomycosis is usually caused by Actinomyces israelii, which is gram-positive, anaerobic bacteria, and normal inhabitants of the human oral cavity $(1,2,4,5,10)$. Actinomycosis is not normally pathogenic, and predisposing conditions include dental caries and extractions, infections, facial trauma, and local tissue damage by surgery $(1,6,7,11)$. There were no medical or dental problems among our patients. However, one person who underwent a facelift procedure is believed to have developed actinomycosis of the parotid gland.

Actinomycosis of the parotid gland appears as a chronic, and slow-growing swelling $(1,7,9,10)$. With the progression of the disease, the development of the fistula tract and discharge occurs $(7,9)$. All the 5 patients complained of swelling and presence of a mass in the parotid gland, and one of them had already developed a fistula at the time of visit. Skin necrosis and fistula tract occurred in the other 2 patients after FNAC (Figure 3). We thought that actinomycosis had progressed to the skin through the FNAC needle passage. 


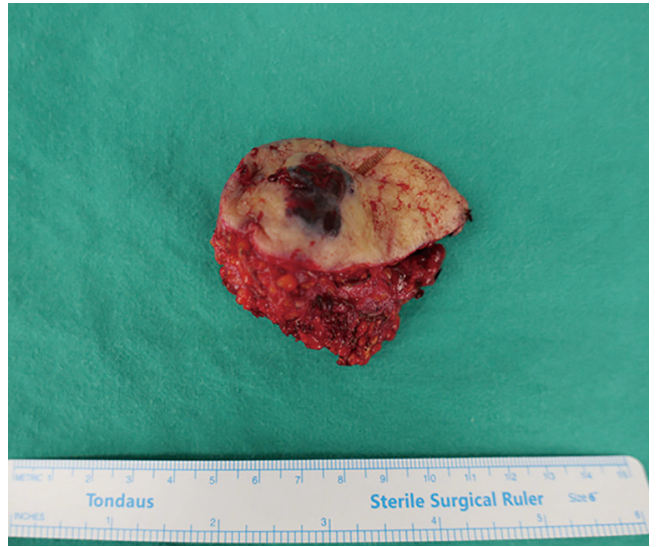

Figure 3 A 72-year-old female patient with actinomycosis of the parotid gland. Surgical specimen with skin necrosis and fistula tract after fine-needle aspiration cytology (FNAC).

It is difficult to differentiate actinomycosis from parotid tumors based on imaging tests including CT, ultrasound (US), magnetic resonance imaging (MRI), and positron emission tomography (PET) $(1,3,5,6,8-11)$. In the present study 4 patients were diagnosed with parotid tumors and 1 patient with parotid abscess before surgery. The definitive diagnosis of actinomycosis of the parotid gland is made by microbiologic or histopathologic findings (1-11). However, the culture of Actinomyces is difficult due to the anaerobic nature of the organism $(4,5,10)$. The diagnosis of Actinomyces in all 3 patients who underwent culture could not be achieved. FNAC or surgical tissue biopsy is essential for accurate diagnosis (1-11). FNAC is known as an easy, safe, and rapid procedure $(1,2)$. However, all four patients who underwent FNAC exhibited failure in the diagnosis of actinomycosis. Consequently, in the absence of clinical and radiological differentiation, and lack of FNAC based diagnosis of actinomycosis from the parotid tumor, it is necessary to perform surgery based on the location of parotid tumors for accurate diagnosis and treatment $(3,5,9)$.

Treatment for actinomycosis of the parotid gland involves antibiotic therapy and surgery (1-11). Penicillin is the drug of choice, but doxycycline, erythromycin, and cephalosporin may be used $(1,4,5,7,9,10)$. However, recommended treatment regimens vary from two weeks to several months $(1,4,5,10)$. Recently, the duration or dose of antibiotics therapy has been determined according to the severity of the disease and the response to treatment $(1,10,11)$. We determined the duration or dose of antibiotic treatment in consultation with the Department of Infectious Medicine after checking the severity of the disease and the patient's symptoms. In the present study, 6 days of intravenous antibiotics administration (on an average) and prescription of oral antibiotics for about 2 months were chosen as the preferred modes of treatment. Surgery for actinomycosis of the parotid glands is mandatory, in cases of extensive abscess, necrotic tissue, fistula tract, or absence of response to antibiotic therapy, and lack of differentiation from parotid tumors $(1,4,5,9-11)$. Four patients with suspicion of parotid tumors underwent superficial parotidectomy before surgery with a safety margin like parotid tumors. One patient with plausible parotid abscess underwent incision and drainage.

The prognosis for actinomycosis of the parotid gland is very good and exhibits improvement in most of the cases (1). All our patients demonstrated improvement with surgery and antibiotic therapy. However, based on the case of one patient with relapse two years after the first treatment, it is proposed that the clinical response should be closely monitored to prevent local recurrence (10).

The limitation of this study is that it is a retrospective study and the number of cases is small.

\section{Conclusions}

Actinomycosis of the parotid gland is very rare, and it is difficult to distinguish it from the parotid tumors based on clinical features or radiologic examinations. In case of skin necrosis of the parotid lesion without evidence of a malignant tumor, or if cancerous cells are not identified in FNAC and fistula tract or skin necrosis occurs after the FNAC, actinomycosis of the parotid gland should be suspected. If the actinomycosis of the parotid gland is localized to the parotid gland and surgically removed, it would be sufficient to treat the patients with antibiotics for about 2 months while observing the clinical response.

\section{Acknowledgments}

Funding: None.

\section{Footnote}

Reporting Checklist: The authors have completed the AME Case Series Checklist. Available at http://dx.doi. org/10.21037/apm-21-755

Data Sharing Statement: Available at http://dx.doi. 
org/10.21037/apm-21-755

Peer Review File: Available at http://dx.doi.org/10.21037/ apm-21-755

Conflicts of Interest: All authors have completed the ICMJE uniform disclosure form (available at http://dx.doi. org/10.21037/apm-21-755). The authors have no conflicts of interest to declare.

Ethical Statement: The authors are accountable for all aspects of the work in ensuring that questions related to the accuracy or integrity of any part of the work are appropriately investigated and resolved. All patient samples were treated in accordance with the Declaration of Helsinki (as revised in 2013), and the study was approved by the independent ethics committees at Chonnam National University Hwasun Hospital (CNUHH-2020-141). And written informed consent was obtained from the patient.

Open Access Statement: This is an Open Access article distributed in accordance with the Creative Commons Attribution-NonCommercial-NoDerivs 4.0 International License (CC BY-NC-ND 4.0), which permits the noncommercial replication and distribution of the article with the strict proviso that no changes or edits are made and the original work is properly cited (including links to both the formal publication through the relevant DOI and the license). See: https://creativecommons.org/licenses/by-nc-nd/4.0/.

\section{References}

1. Oostman O, Smego RA. Cervicofacial Actinomycosis:

Cite this article as: Lee DH, Jung EK, Lee JK, Lim SC. Actinomycosis of the parotid gland: case series. Ann Palliat Med 2021;10(6):6062-6066. doi: 10.21037/apm-21-755
Diagnosis and Management. Curr Infect Dis Rep 2005;7:170-4.

2. Nayak L, DiMaio M, Jeffrey RB. Extraglandular extension of parotid actinomycosis after sonographically guided fineneedle aspiration. J Ultrasound Med 2013;32:715-6.

3. Varghese BT, Sebastian P, Ramachandran K, et al. Actinomycosis of the parotid masquerading as malignant neoplasm. BMC Cancer 2004;4:7.

4. Sittitrai P, Srivanitchapoom C, Pattarasakulchai T, et al. Actinomycosis presenting as a parotid tumor. Auris Nasus Larynx 2012;39:241-3.

5. Moghimi M, Salentijn E, Debets-Ossenkop Y, et al. Treatment of cervicofacial actinomycosis: a report of 19 cases and review of literature. Med Oral Patol Oral Cir Bucal 2013;18:e627-32.

6. Ulvi BM. Actinomycosis of the parotid gland. Plast Reconstr Surg 2005;115:659-61.

7. Dubey A, Murthy JG, Banerjee PK. Actinomycosis of the parotid gland. Indian J Otolaryngol Head Neck Surg 2004;56:306-8.

8. Mok GS, Choi FP, Chu WC. Actinomycosis imitating parotid cancer with metastatic lymph nodes in FDG PET/ CT. Clin Nucl Med 2011;36:309-10.

9. Mamais C, Dias A, Walker J, et al. Parotid actinomycosis mimicking metastatic lymphadenopathy. West Indian Med J 2011;60:349-50.

10. Lan MC, Huang TY, Lin TY, et al. Pathology quiz case 1. Actinomycosis of the lip mimicking minor salivary gland tumor. Arch Otolaryngol Head Neck Surg 2007;133:411-4.

11. Stewart AE, Palma JR, Amsberry JK. Cervicofacial actinomycosis. Otolaryngol Head Neck Surg 2005;132:957-9. 